\title{
COVID-19-associated-mucormycosis: possible role of free iron uptake and immunosuppression
}

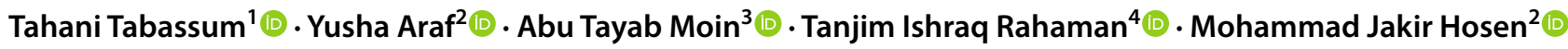

Received: 10 September 2021 / Accepted: 20 October 2021 / Published online: 28 October 2021

(c) The Author(s), under exclusive licence to Springer Nature B.V. 2021

\begin{abstract}
COVID-19-associated-mucormycosis, commonly referred to as the "Black Fungus," is a rare secondary fungal infection in COVID-19 patients prompted by a group of mucor molds. Association of this rare fungal infection with SARS-CoV-2 infection has been declared as an endemic in India, with minor cases in several other countries around the globe. Although the fungal infection is not contagious like the viral infection, the causative fungal agent is omnipresent. Infection displays an overall mortality rate of around 50\%, with many other secondary side effects posing a potential threat in exacerbating COVID-19 mortality rates. In this review, we have accessed the role of free iron availability in COVID-19 patients that might correlate to the pathogenesis of the causative fungal agent. Besides, we have analyzed the negative consequences of using immunosuppressive drugs in encouraging this opportunistic fungal infection.
\end{abstract}

Keywords Mucormycosis $\cdot$ COVID-19 $\cdot$ Rhizopus oryzae $\cdot$ Free iron $\cdot$ Hyperferritinemia $\cdot$ Pathogenesis

\section{Introduction}

The pandemic coronavirus disease (COVID-19), caused by the highly contagious severe acute respiratory syndrome (SARS-CoV-2) virus, has had a catastrophic effect on the

Tahani Tabassum and Yusha Araf contributed equally to this work.

Mohammad Jakir Hosen

jakir-gen@sust.edu

Tahani Tabassum

tahanitabassum0506@gmail.com

Yusha Araf

yusha.araf@gmail.com

1 Biotechnology Program, Department of Mathematics and Natural Sciences, School of Data and Sciences, Brac University, Dhaka, Bangladesh

2 Department of Genetic Engineering and Biotechnology, School of Life Sciences, Shahjalal University of Science and Technology, Sylhet, Bangladesh

3 Department of Genetic Engineering and Biotechnology, Faculty of Biological Sciences, University of Chittagong, Chattogram, Bangladesh

4 Department of Biotechnology and Genetic Engineering, Faculty of Life Sciences, Bangabandhu Sheikh Mujibur Rahman Science and Technology University, Gopalganj, Bangladesh world demographics, resulting in more than 4.8 million deaths worldwide [1]. Even though substantial clinical advancements have been ascertained, the second wave of the virus has recently wreaked havoc on several countries, emerging as the most consistent and consequential global health crisis. Recently, some countries, prominently India, have reported overwhelming numbers of deadly secondary fungal infections in COVID-19 patients. As of May 25, India had reported around 11,700 cases of COVID-19-associatedmucormycosis and declared this disease as an endemic [2], while minor cases of this infection have also been reported in Pakistan Bangladesh, Nepal, Russia, Uruguay, Chile, and Iran [3-9]. The secondary fungal infection usually is rare in immunocompetent hosts. However, it can be deadly if the fungal agent invades immunocompromised hosts or patients with pre-occurring comorbid conditions that increase their susceptibility to such opportunistic infections [10].

Moreover, some COVID-19 patients are currently being treated with steroids or prescribed broad-spectrum antibiotics. Such medicaments are known further to weaken the immune defense mechanism of the patient, increasing susceptibility to other secondary infections $[10,11]$. The exact pathogenesis of this fungal infection in COVID-19 patients is yet explored and needs to be accessed to undertake any potential crisis that the disease might inflict. 


\section{Black fungus and its causative agent}

Mucormycosis is a rare, aggressive, opportunistic infection caused by the group of mucor molds "mucoromycetes" belonging to the order "Mucorales" [12]. The most common causative agents for the infection are the Rhizopus, Lichtheimia, Apophysomyces, Mucor, and Rhizomucor species, whereas occasionally is caused by Cunninghamella and Saksenaea species [13]. The deadly fungal infection can be categorized based on the involvement of the gastrointestinal system, cerebral, nervous system, urogenital system, or the skin. However, the fatal category is the rhino-orbital-cerebral (ROC) and pulmonary involvements [10].

Currently, acute invasive cases of ROC mucormycosis have been reported in COVID-19 patients, and this association between two infections is referred to as the "black fungus." The typical clinical manifestations of this infection are necrosis of the palate or paranasal sinuses that approaches the intra-cranial structures generating symptoms like swelling of the face, nasal and sinus congestion, headache, facial pain, visual disturbances, and black lesion on the nasal bridge or upper part of the mouth that deciphers the name black fungus [14].

Among the family Mucoraceae, the Rhizopus oryzae is the most common agent responsible for nearly $90 \%$ of ROC mucormycosis in humans. This rapidly growing saprophytic filamentous fungus is ubiquitous in the environment and demonstrates an overall mortality rate of $50 \%[10,15]$. The fungi promptly release many sporangiospores into the atmosphere and primarily enter the human body through the respiratory system [12]. Besides, it can be ingested through swallowing spores associated with rotten contaminated organic food or through open wounds. However, these routes of ingestion are rare. Nevertheless, the inhalation of the sporangiospores does not cause disease within immunocompetent hosts, as the host neutrophils and phagocytes generate oxidative metabolites and cationic peptides to inhibit fungal spore proliferation [16-18]. In immunocompromised individuals, especially in cases of hyperglycemia and low $\mathrm{pH}$, phagocytes are rendered dysfunctional in terms of intracellular killing and chemotaxis, enhancing susceptibility to this deadly fungal infection [19].

\section{Pathogenesis of Rhizopus oryzae}

Interactions between the $R$. oryzae and vascular endothelial cells are prominent in fungal pathogenesis. Following paranasal inhalation, $R$. oryzae spores germinate into coenocytic hyphae that initially proliferate in the sinuses and disseminate to the orbit and the brain, primarily through angioinvasion [20]. The fungal spores specifically adhere to the laminin and type IV collagen on the extracellular protein matrixes of the basement membranes separating endothelial cells from underlying stroma [21]. Recently, the glucose-regulated protein 78 (GRP78) receptor was involved in the fungal penetration of endothelial cells [22]. This adherence with the endothelial cells lining the blood vessels mediates fungus endocytosis that facilitates angioinvasion, resulting in vessel thrombosis, hematogenous dissemination, and subsequent tissue necrosis [23]. Another recent transcriptome analysis shows activation of the platelet-derived growth factor (PDGF) pathway in $R$. oryzae pathology, suggesting that PDGF receptors might also facilitate the fungal endothelial invasion [24] and subsequent host cellular injury.

\section{Role of iron in R. oryzae pathology}

One of the virulence factors that enable Mucorales to cause disease is acquiring iron from the host [20]. Iron is a crucial micronutrient that is indispensable for fungal metabolic processes, cell growth, and development. The fungus $R$. oryzae has demonstrated poor growth in normal serum unless an exogenous iron source is provided [25, 26]. Similarly, iron starvation strategies are reported to induce apoptosis in $R$. oryzae [27]. In mammalian hosts, iron sequestration within carrier proteins such as transferrin, ferritin, lactoferrin limits the availability of free iron as a natural strategic defense mechanism against $R$. oryzae $[25,26]$.

Regardless, the fungus has several unique iron-assimilation mechanisms; one prominent mechanism is the release of high-affinity iron permeases (Fig. 1) [28]. These highaffinity iron permeases are part of an integral reductive system, constituting redundant surface reductases that reduce the ferric ion into a more soluble ferrous form. Part of this reductive system is a copper oxidase-ferrous permease complex that captures those soluble ferrous ions, making those available for fungal acquisition (Fig. 1) [28-30]. Recent data shows the gene encoding high-affinity iron permease (FTR1) is overexpressed by $R$. oryzae during murine infection, implying the role of such permeases in enhancing fungal iron uptake [31]. Besides, the fungus may secrete siderophores, low molecular weight iron chelators, such as rhizoferrin, or utilize xenosiderophores such as Deferoxamine that enhances iron uptake (Fig. 1) [20,32]. Genomic analysis of $R$. oryzae identified 13 siderophore permeases that might serve as siderophore receptors [33]. Rhizoferrin may supply iron to the $R$. oryzae through an energy-dependent receptormediated pathway, but the exact mechanism of whether this siderophore releases iron extracellularly or is internalized 


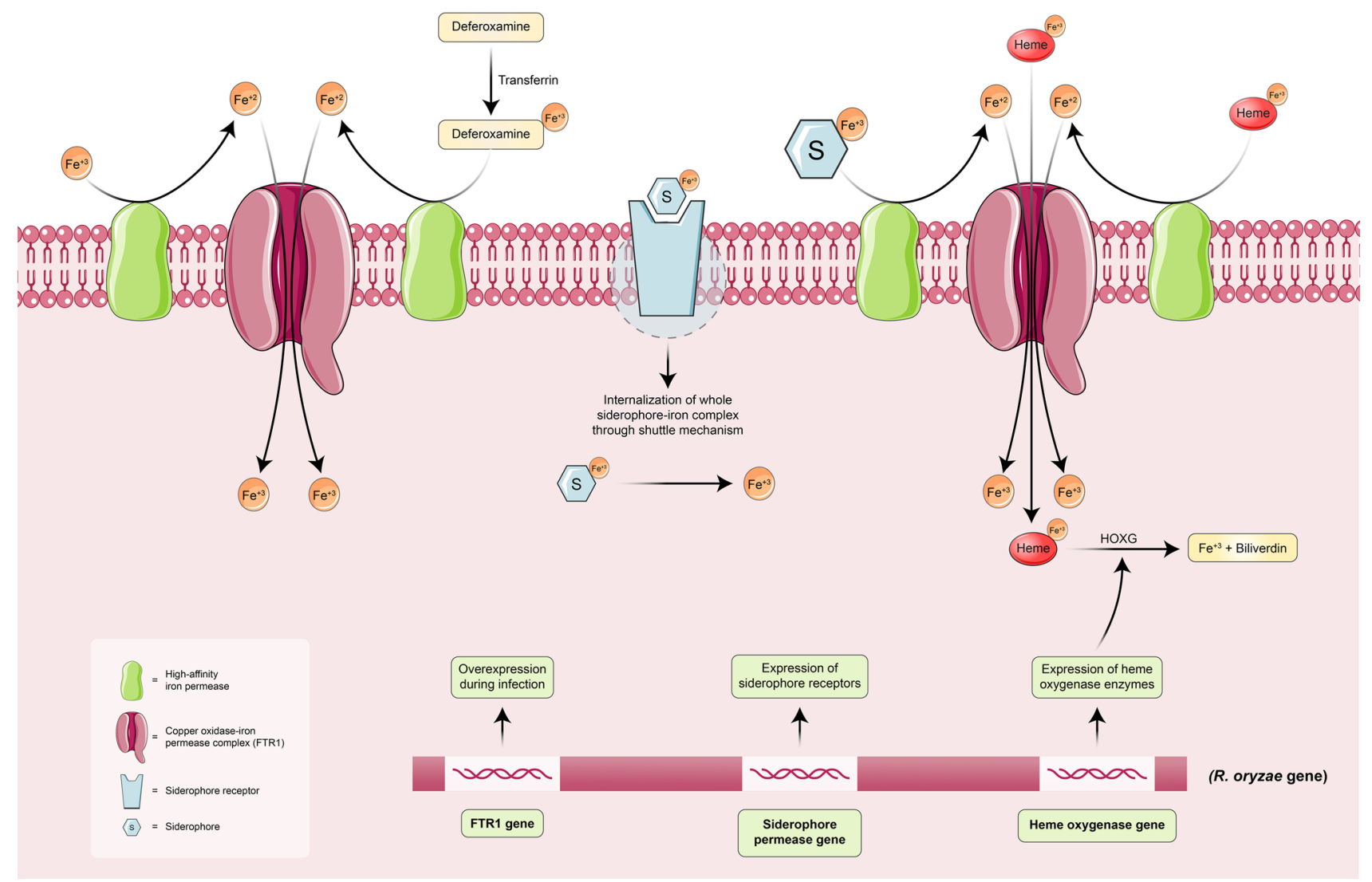

Fig. 1 Different mechanisms utilized by Mucorales to obtain iron from the host. High-affinity iron permeases on the cytoplasmic membrane can convert the less soluble ferric ion into more soluble ferrous ions, followed by their internalization through the copper oxidaseiron permease (FTR1) complex. The complex oxidizes the ferrous form into the ferric form required to properly utilize the ion in intracellular processes. A xenosiderophore, Deferoxamine, may strip $\mathrm{Fe}^{3+}$ ion from host transferrin and produce ferrioxamine (Deferoxamine$\mathrm{Fe}^{3+}$ complex) that is reduced into ferrous ion and internalized by FTR1 complex. Other endogenous siderophores are synthesized by the fungus chelate iron extracellularly, and the whole complex may be

before releasing iron is not known yet [32, 34]. Two more recently introduced iron chelators are Deferiprone (act as bidentate chelator) and Deferasirox (act as tridentate chelator). Although both of these chelators have displayed significant depletion of iron levels in clinical practices [35-37], these have not been reported to increase susceptibility to mucormycosis [38, 39]. Besides, these two new chelators do not act as xenosiderophores as the fungal iron uptake system cannot detach iron from them. Their higher affinity for iron forms much stable chemical structure indifferent to fungal iron uptake systems. Another reason might be the lower molecular masses of these chelators limit their access to fungal iron uptake systems [39].

Moreover, the genomic analysis of $R$. oryzae revealed two homologs of heme oxygenase [33], enabling the fungus to obtain iron from host hemoglobin and explain their internalized through a shuttle mechanism. Alternatively, the siderophore-iron complexes may be reduced through membrane permeases to generate $\mathrm{Fe}^{2+}$ ions, followed by internalization by the FTR1 complex. The angioinvasive nature of the infection also reveals that heme can be a potential source of iron. The heme- $\mathrm{Fe}^{3+}$ complex may be entirely internalized and then acted on by the heme oxygenases intracellularly or reduced on the surface to generate $\mathrm{Fe}^{2+}$ ions to be taken in by the FTR1 complex. Overexpression of the FTR1 gene during infection correlates to the requirement of this complex in internalizing all the extracellular ferrous ions and some iron chelator complexes

angioinvasive nature. Genomic analysis of $R$. oryzae depicts a reduced efficiency of utilizing heme as an iron source when the copy number of the FTRI gene is reduced [31], suggesting that FTR1 may serve as a membrane permease enabling uptake of extracellular heme and its subsequent intracellular degradation through heme oxygenases to generate free iron (Fig. 1).

\section{Correlation with COVID pathology}

\section{Generation of free iron}

Hyperferritinemia is a crucial diagnostic and prognostic laboratory biomarker in COVID-19 infection [40-45]. Increased serum ferritin is a common manifestation in 
COVID-19 inflammatory reactions and can also contribute to the development of cytokine storms [46]. Hyperferritinemia and inflammation are the primary mediators of COVID19 associated dysregulation of iron homeostasis [47]. Apart from being an active secretion during inflammation, hyperferritinemia induces hepatic cell death [48], and apoptosis releases the intracellularly stored free irons and serum ferritin to the cell exterior. The stored serum ferritin from hepatocytes on release loses part of the inner iron content, releasing extremely high levels of free iron (Fig. 2) [49].

Consequently, the high incidence of hyperferritinemia is consistent with the emergence of enhanced levels of free iron in COVID-19 patients [50] that R. oryzae might utilize for fungal proliferation and growth. Similarly, inflammationinduced imbalance of iron regulatory hormone hepcidin may elevate free iron levels in COVID-19 patients (Fig. 2) [51]. Another key pathogenic strategy of SARS-CoV-2 that might ensure iron availability for $R$. oryzae pathogenesis is to attack the 1-beta chain of hemoglobin that initiates dissociation of porphyrins from iron and rapid discharge of elevated amounts of iron into circulation (Fig. 2) [52].

\section{Suppression of the immune system}

Although the host immune system plays a crucial role in SARS-CoV-2 virus suppression, the excessive release of

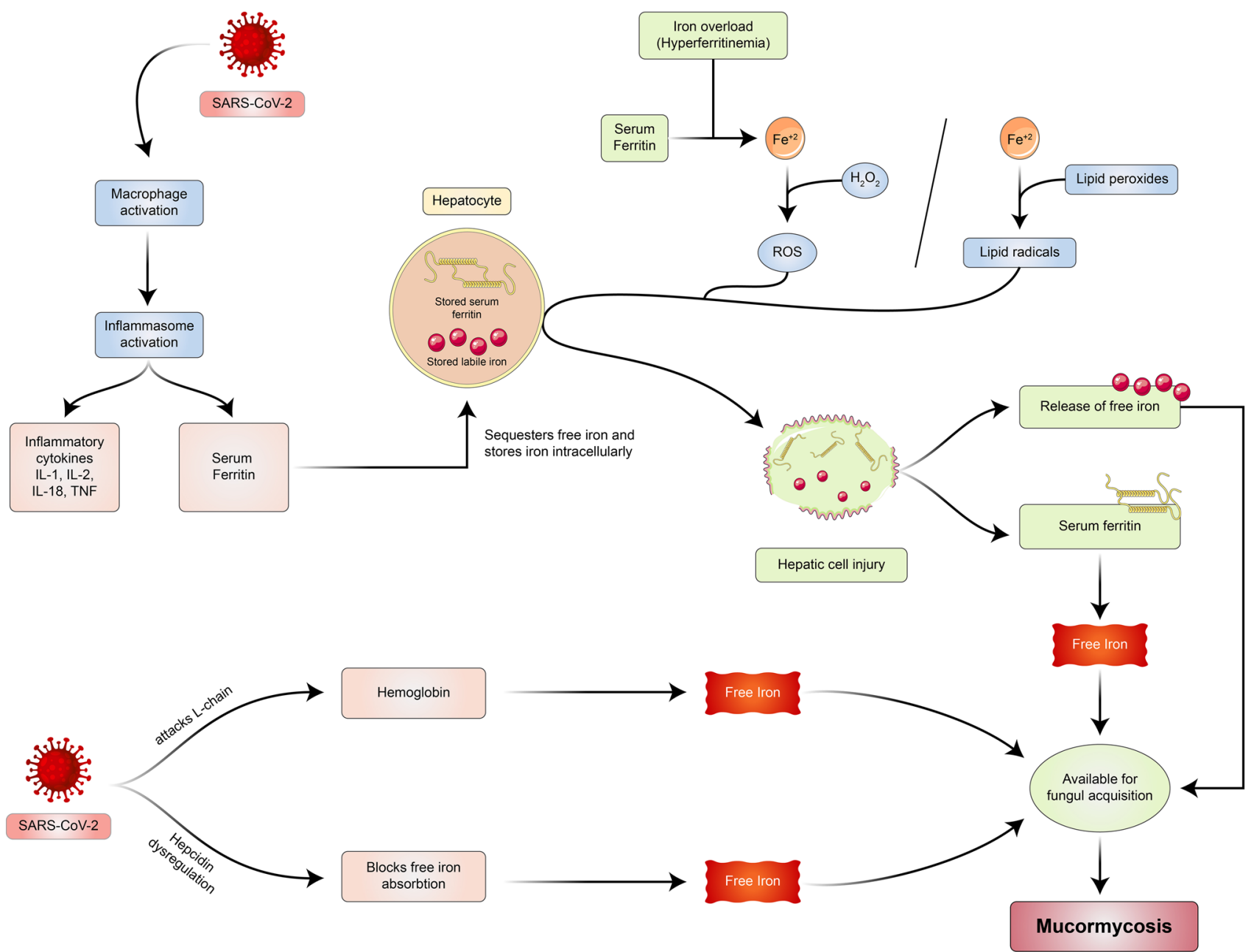

Fig. 2 Higher levels of free iron accumulation during COVID-19 infection. The SARS-CoV-2 virus displays hyperferritinemic syndrome (hyperferritinemia and hyperinflammation). The viral infection activates macrophages, which stimulates the release of elevated cytokine content and serum ferritin. Serum ferritin is advantageous to block secondary fungal infection as it sequesters free iron, resulting in iron starvation for Mucorales. During iron overload, like hyperferritinemia generated in COVID-19 infection, serum ferritin stored in hepatocyte undergoes denaturation in the lysosome, and the $\mathrm{Fe}^{2+}$ bound to ferritin is released. The labile $\mathrm{Fe}^{2+}$ reacts with hydrogen peroxide $\left(\mathrm{H}_{2} \mathrm{O}_{2}\right)$ to generate highly toxic reactive oxygen species (ROS) or lipid peroxides to generate lipid radicals, which induces hepatic cell death and releases the stored labile iron and serum ferritin extracellularly. Moreover, the SARS-CoV-2 virus may attack hemoglobin or induce hepcidin dysregulation, the host's major iron homeostasis regulation hormone. All of these marks free iron availability for fungal acquisition, facilitating mucormycosis in COVID-19 patients 
inflammatory and pro-inflammatory mediators aggravates the cytokine storm. It exacerbates COVID-19 induced damage to the host organs [53]. Consequently, several immunosuppressive drugs have become promising agents in treating severe COVID-19 cases, including steroids, anti-cytokine agents, mTOR (mammalian target of Rapamycin) inhibitors, and antimetabolites [54]. These immunosuppressive agents may weaken the patients' immune system, enabling secondary opportunistic infections, including mucormycosis. Besides, steroids are the main reason for drug-induced hyperglycemia [55], one of the significant risk factors for mucormycosis [56].

Moreover, the COVID-19 pathogenesis itself may trigger immune-deprived conditions by entering the immune cells for utilizing its metabolic machinery for viral replication and eliciting subsequent destruction of the immune cells [57]. The viral infection may generate complex immune dysregulation and suppress the immune defense system [58, 59]. Such immune suppression mediated by the virus itself may increase the susceptibility to secondary opportunistic infections, including $R$. oryzae associated mucormycosis.

\section{Conclusion}

Rhino-orbital-cerebral mucormycosis is emerging as a deadly secondary infection in COVID-19 that can rapidly upsurge the mortality rates and develop into another significant global concern. This aggressive, opportunistic fungal infection not only displays an overall mortality rate of 50\% but also provokes other disabling side effects such as visual impairment or eye loss, which might significantly add to the crippling global debridement this pandemic has incurred on human lives.

Excessive free iron levels are accessible to be utilized by Mucorales and other potential pathogens with chances of creating secondary infection in COVID-19 patients and can lead to fibrosis through redox reaction [60]. Since free iron appears to play a significant role in encouraging fungal proliferation and growth, it is hypothesized that iron depletion or chelation therapy in COVID-19 patients might positively impact controlling this fungal infection. Additionally, iron depletion therapy might also represent a promising therapeutic approach against SARS-CoV-2 replication itself, as studies have demonstrated a positive association between free iron availability and a worse prognosis of other viral infections [61].

The aggressive mycotic ROC mucormycosis infection has a high mortality rate; however, early diagnosis and treatment might facilitate curtailing the mortality rate in COVID19 patients to some extent. Diagnosis of black fungus is most popularly reliant on the typical clinical presentations such as blackish nasal mucous emission, sinusitis, visual impairment, swelling and pain on only one side of the face with loss of sensation, the node of the orbit, black lesions around the nasal bridge, proptosis, abnormal blood clotting and thrombosis of tissues, and headache. On endoscopic visualization, there might be appearances of dead black tissue mass around the nasal cavity that can be seen even on opening the mouth $[11,62,63]$. Based on clinical suspicion, more specified diagnosis tests such as MRI and CT scan of the nasal cavity, sinuses, or brain are performed to determine lesions' presence and extent [62]. Additionally, histopathological examination of biopsy samples and culturing of the fungal agent can be performed to detect the causative fungal agent [64].

One of the standard features in ROC mucormycosis is the accumulation of fungal debris in the oropharyngeal region that might initiate difficulty in breathing [65]. Besides, COVID-19 patients infected by the fungus may exhibit rapid respiratory function deterioration, including the build-up of excess fluid in the lungs [63]. Since COVID-19 infection also reveals similar patterns of respiratory difficulty, it might be challenging to distinguish the presence of the fungal infection within COVID-19 patients. Upon examining such symptoms, definitive tests must be performed to identify the pathogenic agent through sample culturing.

The group of COVID-19 patients who are more susceptible to mucormycosis, such as patients with diabetes (especially diabetes ketoacidosis), cancer, organ transplant, stem cell transplant, neutropenia, long-term use of steroids, hemochromatosis, skin injury $[10,11,62,66]$, and immunocompromised, must be taken under special consideration. Such comorbid conditions impair the immune defense function, making the patient more prone to getting affected by the fungal agent [63]. Besides, COVID-19 patients who are already taking prescribed broad-spectrum antibiotics or antifungal drugs to combat infections must be brought under special consideration [63], as broad-spectrum antibiotics often disrupt the beneficial microflora and increase the susceptibility of getting attacked by opportunistic infections.

COVID-19 patients with comorbid conditions that stimulate mechanisms to generate iron overload disorder, such as hemochromatosis and beta-thalassemia [67, 68], are at greater risk of getting attacked by the fungus-free iron accumulates within their body. Such patients must be kept in special monitoring to reduce any chances of getting affected by the fungus. Moreover, immunosuppressive drugs, especially steroids, must be regulated appropriately, and proper maintenance and monitoring of blood sugar levels. Patients already taking prescribed steroid medicaments for preoccurring conditions must be taken special care of due to their weakened immune system and higher chances of getting attacked by the fungus. The appropriate doses and duration of the steroid should be ensured during the treatment and after recovery [69]. Additional steps to enhance their 
immune system against this opportunistic infection must also be ensured.

Healthcare practitioners can play a vital role in reducing cases of fungal infection by encouraging the patients to practice safe health and hygiene. Since the fungus is ubiquitously suspended throughout the environment, patients who demonstrate COVID-19 symptoms, those who have recovered from covid-19, and those with compromised immune systems must be aware of the situation. Especially, COVID-19 patients with comorbid conditions that enhance susceptibility to this fungal infection must be cautioned by the healthcare practitioners to practice safe hygiene. Patients with prescribed antibiotics and steroids must also be monitored thoroughly, and the exhibition of any symptoms associated with black fungus must be adequately examined. Healthcare practitioners must be cautious in prescribing antibiotics and steroids throughout the pandemic period while following proper guidelines. Moreover, the testing facilities for the COVID-19 patients must also ensure sterility protocols as experts have suggested that RT-PCR tests that rely on cotton swabs being inserted within the nasal passage of patients might be one of the sources of the fungal infection if the process is not conducted with ensured sterility [70]. However, the association between the two infections is not wellstudied, but extensive laboratory studies have to be initiated to unleash the disease pathogenesis and their correlations completely.

Acknowledgements The authors acknowledge the members of the Community of Biotechnology, Dhaka, Bangladesh, for their support during the preparation of the manuscript.

Author contributions YA conceived the study. YA, TT, AM designed the study. TT and YA wrote the draft manuscript. TT, YA, and MH carried out the revisions. All authors approved the final version of the manuscript.

Funding The authors received no funding from external sources.

\section{Declarations}

Conflict of interest The authors declare that they have no known competing financial interests or personal relationships that could have influenced the work reported in this paper.

\section{References}

1. COVID live update: $172,045,061$ cases and 3,578,246 deaths from the coronavirus - worldometer. Worldometers.info. 2021. https:// www.worldometers.info/coronavirus/. Accessed 2 June 2021

2. Sensharma A (2021) Amid COVID-19 pandemic, India reports over 11,700 black fungus cases I Check state-wise report on mucormycosis cases here. Jagran English. https://english.jagran. com/india/amid-covid19-pandemic-india-reports-over-11700black-fungus-cases-check-statewise-report-on-mucormycosiscases-here-10027224. Accessed 2 June 2021
3. Bhatti M (2021) 'Cases of black fungus emerge across Pakistan'. The News International. https://www.thenews.com.pk/amp/ 834117-cases-of-black-fungus-emerge-across-pakistan. Accessed 2 June 2021

4. Molla M (2021) Two black fungus cases detected in Bangladesh. The Daily Star. https://www.google.com/amp/s/www.thedailyst ar.net/bangladesh/news/two-black-fungus-cases-detected-20983 09\%3famp. Accessed 2 June 2021

5. Zykov K (2021) Russia confirms rare, deadly 'black fungus' infections seen in India - Reports - The Moscow Times. The Moscow Times. https://www.themoscowtimes.com/2021/05/17/russiaconfirms-rare-deadly-black-fungus-infections-seen-in-india-repor ts-a73922. Accessed 2 June 2021

6. Focused COVID-19 Media Monitoring, Nepal (May 24, 2021) Nepal. ReliefWeb (2021). https://reliefweb.int/report/nepal/focus ed-covid-19-media-monitoring-nepal-may-24-2021. Accessed 2 June 2021

7. Urwicz T (2021) Paciente con COVID-19 se infectó con el "hongo negro". Diario EL PAIS Uruguay. https://www.elpais.com.uy/ informacion/salud/paciente-covid-infecto-hongo-negro.html?_. Accessed 2 June 2021

8. Mostrador E (2021) Detectan primer caso de "hongo negro" en Chile en paciente con Covid-19: es el segundo reportado en Latinoamérica. El Mostrador. https://www.elmostrador.cl/dia/2021/ 05/28/detectan-primer-caso-de-hongo-negro-en-chile-en-pacie nte-con-covid-19-es-el-segundo-reportado-en-latinoamerica/. Accessed 2 June 2021

9. Coronavirus in Iran: power outages, black fungus, and warnings of a fifth surge - Track Persia. Track Persia (2021). https://www. trackpersia.com/coronavirus-in-iran-power-outages-black-fungusand-warnings-of-a-fifth-surge/. Accessed 2 June 2021

10. Roden M, Zaoutis T, Buchanan W, Knudsen T, Sarkisova T, Schaufele R et al (2005) Epidemiology and outcome of zygomycosis: a review of 929 reported cases. Clin Infect Dis 41(5):634-653

11. Mucormycosis/black fungus: why are COVID-19 patients at risk? Here are ICMR guidelines for prevention. Netmeds (2021). https:// www.netmeds.com/health-library/post/mucormycosis-black-fungus-why-are-covid-19-patients-at-risk-here-are-icmr-guidelinesfor-prevention. Accessed 4 June 2021

12. Bhatt K, Agolli AH, Patel M, Garimella R, Devi M, Garcia E et al (2021) High mortality co-infections of COVID-19 patients: mucormycosis and other fungal infections. Discoveries 9(1):126.

13. AK AK, Gupta V (2021 Jan) Rhino-orbital cerebral mucormycosis. [Updated 2021 May 1]. In: StatPearls. Treasure Island (FL): StatPearls Publishing. https://www.ncbi.nlm.nih.gov/books/ NBK557429/

14. Yohai R, Bullock J, Aziz A, Markert R (1994) Survival factors in rhino-orbital-cerebral mucormycosis. Surv Ophthalmol 39(1):3-22

15. Ribes J, Vanover-Sams C, Baker D (2000) Zygomycetes in human disease. Clin Microbiol Rev 13(2):236-301

16. Waldorf A, Ruderman N, Diamond R (1984) Specific susceptibility to mucormycosis in murine diabetes and bronchoalveolar macrophage defense against Rhizopus. J Clin Investig 74(1):150-160

17. Waldorf AR (1989) Pulmonary defense mechanisms against opportunistic fungal pathogens. Immunol Ser 47:243-271

18. Diamond R, Haudenschild C, Erickson N (1982) Monocyte-mediated damage to Rhizopus oryzae hyphae in vitro. Infect Immunity 38(1):292-297

19. Chinn R, Diamond R (1982) Generation of chemotactic factors by Rhizopus oryzae in the presence and absence of serum: relationship to hyphal damage mediated by human neutrophils and effects of hyperglycemia and ketoacidosis. Infect Immunity 38(3):1123-1129

20. Ibrahim A, Spellberg B, Walsh T, Kontoyiannis D (2012) Pathogenesis of mucormycosis. Clin Infect Dis 54(suppl_1):S16-S22 
21. Bouchara JP, Oumeziane NA, Lissitzky JC, Larcher G, Tronchin G, Chabasse D (1996) Attachment of spores of the human pathogenic fungus Rhizopus oryzae to extracellular matrix components. Eur J Cell Biol 70(1):76-83

22. Liu M, Spellberg B, Phan Q, Fu Y, Fu Y, Lee A et al (2010) The endothelial cell receptor GRP78 is required for mucormycosis pathogenesis in diabetic mice. J Clin Investig 120(6):1914-1924

23. Ibrahim A, Spellberg B, Avanessian V, Fu Y, Edwards J (2005) Rhizopus oryzae adheres to, is phagocytosed by, and damages endothelial cells in vitro. Infect Immunity 73(2):778-783

24. Chibucos M, Soliman S, Gebremariam T, Lee H, Daugherty S, Orvis J et al (2016) An integrated genomic and transcriptomic survey of mucormycosis-causing fungi. Nat Commun 7(1):1-11

25. Artis W, Fountain J, Delcher H, Jones H (1982) A mechanism of susceptibility to mucormycosis in diabetic ketoacidosis transferrin and iron availability. Diabetes 31(12):1109-1114

26. Boelaert J, de Locht M, Van Cutsem J, Kerrels V, Cantinieaux B, Verdonck A et al (1993) Mucormycosis during deferoxamine therapy is a siderophore-mediated infection. In vitro and in vivo animal studies. J Clin Investig 91(5):1979-1986

27. Shirazi F, Kontoyiannis D, Ibrahim A (2015) Iron starvation induces apoptosis in Rhizopus oryzae in vitro. Virulence 6(2):121-126

28. Stearman R, Yuan D, Yamaguchi-Iwai Y, Klausner R, Dancis A (1996) A permease-oxidase complex involved in high-affinity iron uptake in yeast. Science 271(5255):1552-1557

29. Knight S, Vilaire G, Lesuisse E, Dancis A (2005) Iron acquisition from transferrin by Candida albicans depends on the reductive pathway. Infect Immunity 73(9):5482-5492

30. Jung W, Sham A, Lian T, Singh A, Kosman D, Kronstad J (2008) Iron source preference and regulation of iron uptake in Cryptococcus neoformans. PLoS Pathog 4(2):e45

31. Ibrahim A, Gebremariam T, Lin L, Luo G, Husseiny M, Skory C et al (2010) The high affinity iron permease is a key virulence factor required for Rhizopus oryzae pathogenesis. Mol Microbiol 77(3):587-604

32. Thieken A, Winkelmann G (1992) Rhizoferrin: A complexone type siderophore of the mocorales and entomophthorales (Zygomycetes). FEMS Microbiol Lett 94(1-2):37-41

33. Ma L, Ibrahim A, Skory C, Grabherr M, Burger G, Butler M et al (2009) genomic analysis of the basal lineage fungus Rhizopus oryzae reveals a whole-genome duplication. PLoS Genet 5(7):e1000549

34. De Locht M, Boelaert J, Schneider Y (1994) Iron uptake from ferrioxamine and from ferrirhizoferrin by germinating spores of rhizopus microsporus. Biochem Pharmacol 47(10):1843-1850

35. DivakarJose R, Delhikumar C, Ram KG (2020) Efficacy and safety of combined oral chelation with deferiprone and deferasirox on iron overload in transfusion dependent children with thalassemia: a prospective observational study. Indian J Pediatr 88(4):330-335

36. Lui G, Obeidy P, Ford S, Tselepis C, Sharp D, Jansson P et al (2012) The iron chelator, deferasirox, as a novel strategy for cancer treatment: oral activity against human lung tumor xenografts and molecular mechanism of action. Mol Pharmacol 83(1):179-190

37. Neufeld E (2006) Oral chelators deferasirox and deferiprone for transfusional iron overload in thalassemia major: new data, new questions. Blood 107(9):3436-3441

38. Boelaert J, Van Cutsem J, de Locht M, Schneider Y, Crichton R (1994) Deferoxamine augments growth and pathogenicity of Rhizopus, while hydroxypyridinone chelators have no effect. Kidney Int 45(3):667-671

39. Symeonidis A (2009) The role of iron and iron chelators in zygomycosis. Clin Microbiol Infect 15:26-32
40. Zhou F, Yu T, Du R, Fan G, Liu Y, Liu Z et al (2020) Clinical course and risk factors for mortality of adult inpatients with COVID-19 in Wuhan, China: a retrospective cohort study. Lancet 395(10229):1054-1062

41. Wu C, Chen X, Cai Y, Xia J, Zhou X, Xu S et al (2020) risk factors associated with acute respiratory distress syndrome and death in patients with coronavirus disease 2019 pneumonia in Wuhan, China. JAMA Intern Med 180(7):934

42. Bozkurt F, Tercan M, Patmano G, Bingol Tanriverdi T, Demir H, Yurekli U (2021) Can ferritin levels predict the severity of illness in patients with COVID-19?. Cureus

43. Zhou B, She J, Wang Y, Ma X (2020) Utility of ferritin, procalcitonin, and C-reactive protein in severe patients with 2019 novel coronavirus disease

44. Fox S, Akmatbekov A, Harbert J, Li G, Brown J, Heide R (2020) Pulmonary and cardiac pathology in Covid-19: the first autopsy series from New Orleans

45. Chen N, Zhou M, Dong X, Qu J, Gong F, Han Y et al (2020) Epidemiological and clinical characteristics of 99 cases of 2019 novel coronavirus pneumonia in Wuhan, China: a descriptive study. Lancet 395(10223):507-513

46. Theurl I, Aigner E, Theurl M, Nairz M, Seifert M, Schroll A et al (2009) Regulation of iron homeostasis in anemia of chronic disease and iron deficiency anemia: diagnostic and therapeutic implications. Blood 113(21):5277-5286

47. Kernan K, Carcillo J (2017) Hyperferritinemia and inflammation. Int Immunol 29(9):401-409

48. Takami T, Sakaida I (2011) Iron regulation by hepatocytes and free radicals. J Clin Biochem Nutr 48(2):103-106

49. Pretorius E, Kell D (2014) Diagnostic morphology: biophysical indicators for iron-driven inflammatory diseases. Integr Biol 6(5):486-510

50. Edeas M, Saleh J, Peyssonnaux C (2020) Iron: innocent bystander or vicious culprit in COVID-19 pathogenesis? Int J Infect Dis 97:303-305

51. Nemeth E, Rivera S, Gabayan V, Keller C, Taudorf S, Pedersen $B$ et al (2004) IL-6 mediates hypoferremia of inflammation by inducing the synthesis of the iron regulatory hormone hepcidin. J Clin Investig 113(9):1271-1276

52. wenzhong 1, hualan L (2020) COVID-19:attacks the 1-beta chain of hemoglobin and captures the porphyrin to inhibit human heme metabolism

53. Henderson L, Canna S, Schulert G, Volpi S, Lee P, Kernan K et al (2020) On the alert for cytokine storm: immunopathology in COVID -19. Arthritis Rheumatol 72(7):1059-1063

54. Schoot T, Kerckhoffs A, Hilbrands L, van Marum R (2020) Immunosuppressive drugs and COVID-19: a review. Front Pharmacol $11: 1333$

55. van Raalte D, Ouwens D, Diamant M (2009) Novel insights into glucocorticoid-mediated diabetogenic effects: towards expansion of therapeutic options? Eur J Clin Investig 39(2):81-93

56. Spellberg B, Edwards J, Ibrahim A (2005) Novel perspectives on mucormycosis: pathophysiology, presentation, and management. Clin Microbiol Rev 18(3):556-569

57. Wang K, Chen W, Zhou Y, Lian J, Zhang Z, Du P et al (2020) SARS-CoV-2 invades host cells via a novel route: CD147-spike protein

58. Infantino M, Damiani A, Gobbi FL, Grossi V, Lari B, Macchia D (2020) Serological assays for SARS-CoV-2 infectious disease: benefits, limitations and perspectives. Isr Med Assoc J IMAJ 22(4):203-210

59. Chiappelli F (2020) CoViD-19 immunopathology \& immunotherapy. Bioinformation 16(3):219-222

60. Perricone C, Bartoloni E, Bursi R, Cafaro G, Guidelli G, Shoenfeld $\mathrm{Y}$ et al (2020) COVID-19 as part of the hyperferritinemic 
syndromes: the role of iron depletion therapy. Immunol Res 68(4):213-224

61. Liu W, Zhang S, Nekhai S, Liu S (2020) Depriving iron supply to the virus represents a promising adjuvant therapeutic against viral survival. Curr Clin Microbiol Rep 7(2):13-19

62. Petrikkos G, Skiada A, Lortholary O, Roilides E, Walsh T, Kontoyiannis D (2012) Epidemiology and clinical manifestations of mucormycosis. Clin Infect Dis 54(suppl_1):S23-S34

63. Mucormycosis: why are Covid-19 patients being affected by black fungus infection?. The Indian Express (2021). https://indianexpr ess.com/article/lifestyle/health/mucormycosis-why-are-covid-19patients-being-affected-by-black-fungus-7321627/lite/. Accessed 4 June 2021

64. Chen Y, He Y, Zhou H, Wang M, Su S (2017) Rapidly progressive rhino-orbito-cerebral mucormycosis in a patient with type 2 diabetes: a case report. Exp Ther Med 13(3):1054-1056

65. Karaaslan E (2019) Anesthetic management of rhinoorbitocerebral mucormycosis; focus on challenges. J de Mycologie Médicale 29(3):219-222

66. Walsh T, Gamaletsou M, McGinnis M, Hayden R, Kontoyiannis D (2012) Early clinical and laboratory diagnosis of invasive pulmonary, extrapulmonary, and disseminated mucormycosis (Zygomycosis). Clin Infect Dis 54(suppl_1):S55-S60

67. Brazier Y (2020) Iron overload disorder: symptoms, causes, and treatment. Medicalnewstoday.com. https://www.medicalnewstoday.com/articles/166455. Accessed 4 June 2021

68. Mishra AK, Tiwari A (2013) Iron overload in Beta thalassaemia major and intermedia patients. Maedica (Bucur) 8(4):328-332

69. Biswas S (2021) Mucormycosis: the 'black fungus' maiming Covid patients in India. BBC News. https://www.bbc.com/news/worldasia-india-57027829. Accessed 4 June 2021

70. Sharma J (2021) Are nasal swabs used in covid testing behind the outbreak of black fungus? https://www.outlookindia.com/. https://www.outlookindia.com/website/story/india-news-arenasal-swabs-used-in-covid-testing-behind-the-outbreak-of-blackfungus/383370. Accessed 4 June 2021

Publisher's Note Springer Nature remains neutral with regard to jurisdictional claims in published maps and institutional affiliations. 